\title{
An extreme-AO search for giant planets around a white dwarf ${ }^{\star, \star \star}$ VLT/SPHERE performance on a faint target GD 50
}

\author{
S. Xu (许偲艺) ${ }^{1}$, S. Ertel $^{2}$, Z. Wahhaj ${ }^{2,3}$, J. Milli $^{2}$, P. Scicluna ${ }^{4}$, and G. H.-M. Bertrang ${ }^{4}$ \\ ${ }^{1}$ European Southern Observatory, Karl-Schwarzschild-Straße 2, 85748 Garching, Germany \\ e-mail: sxu@eso.org \\ 2 European Southern Observatory, Alonso de Cordova 3107, Vitacura, 19001 Casilla, Santiago 19, Chile \\ 3 Aix-Marseille Université, CNRS, LAM (Laboratoire d'Astrophysique de Marseille) UMR 7326, 13388 Marseille, France \\ ${ }^{4}$ Kiel University, Institute of Theoretical Physics and Astrophysics, Leibnizstr. 15, 24118 Kiel, Germany
}

Received 24 March 2015 / Accepted 15 June 2015

\section{ABSTRACT}

\begin{abstract}
Context. Little is known about the planetary systems around single white dwarfs, although there is strong evidence that they do exist. Aims. We performed a pilot study with the extreme-AO system on the Spectro-Polarimetric High-contrast Exoplanet REsearch (SPHERE) on the Very Large Telescopes (VLT) to look for giant planets around a young white dwarf, GD 50.

Methods. We were awarded science verification time on the new ESO instrument SPHERE. Observations were made with the InfraRed Dual-band Imager and Spectrograph in classical imaging mode in $H$ band.

Results. Despite the faintness of the target (14.2 mag in $R$ band), the AO loop was closed and a strehl of $37 \%$ was reached in $H$ band. No objects were detected around GD 50. We achieved a 5-sigma contrast of 6.2, 8.0, and 8.25 mag at 0.'2, 0.'4, and 0.'6 and beyond, respectively. We exclude any substellar objects more massive than $4.0 M_{J}$ at $6.2 \mathrm{au}, 2.9 M_{J}$ at $12.4 \mathrm{au}$, and $2.8 M_{J}$ at 18.6 au and beyond. This rivals the previous upper limit set by Spitzer. We further show that SPHERE is the most promising instrument available to search for close-in substellar objects around nearby white dwarfs.
\end{abstract}

Key words. planets and satellites: detection - white dwarfs

\section{Introduction}

Radial velocity and transit surveys demonstrate that extrasolar planets are prevalent around main-sequence stars in the Milky Way (Cumming et al. 2008). However, little is known about the evolution of planetary systems beyond the main sequence lifetime of their host stars. Planets have been discovered around (sub)giant stars (Johnson et al. 2011; Gettel et al. 2012) and horizontal branch stars (Silvotti et al. 2007; Setiawan et al. 2010; Charpinet et al. 2011). There is only one planetarymass object detected with direct imaging around a white dwarf (WD 0806-661; Luhman et al. 2011), although theoretical calculations show that many more planets can survive the red giant stage (Burleigh et al. 2002; Jura 2008; Mustill \& Villaver 2012; Nordhaus \& Spiegel 2013).

From an observational perspective, the strongest evidence for planets around single white dwarfs comes from the study of heavy-element-enriched white dwarfs. About $25 \%$ to $50 \%$ of white dwarfs have heavy elements in their atmospheres, in addition to the primordial hydrogen or helium (Zuckerman et al. 2010; Koester et al. 2014). There must be a constant supply of material onto the white dwarf to balance the rapid settling of heavy elements out of the photosphere (Koester 2009). In addition, some of these heavy-element-enriched white dwarfs also display infrared excess from a circumstellar dust disk (Jura et al. 2007; Xu \& Jura 2012) or, more rarely, from a gaseous

\footnotetext{
* Based on observations made with European Southern Observatory (ESO) telescopes at the La Silla Paranal Observatory under program 60.A-9373(A).

$\star \star$ Figure 4 is available in electronic form at http://www . aanda.org
}

disk (Gänsicke et al. 2006). The standard model is that these were formed by asteroids that were perturbed by the surviving planet(s) in the system onto orbits that crossed the tidal radius of the white dwarf, were disrupted, and then formed a dust disk. Eventually, these materials were accreted onto the white dwarf, enriching its atmosphere (Jura 2003; Jura \& Young 2014). This asteroid accretion model is further supported by comparing the abundances of the accreting material with solar system objects: they all resemble terrestrial material rather than the cosmic abundance (Klein et al. 2010; Jura et al. 2012; Gänsicke et al. 2012; Xu et al. 2014).

Dynamical simulations show that there are different pathways that can lead to the asteroid-accretion model. The configuration varies between having a comet belt with two planets, an inner planet and a Neptune-sized planet (Bonsor et al. 2011), an asteroid belt with a Jupiter-sized planet (Debes et al. 2012) and an asteroid belt with an eccentric sub-Jupiter-sized planet (Frewen \& Hansen 2014). At least one planet must be present to explain all the observed features of the system.

However, classical planet-hunting methods do not work well for white dwarfs. The red giant star has cleared planets within a few au, making the transit probability very low. White dwarfs have far too few spectral lines to make accurate radial velocity measurements. Here, we summarize a few previous searches for planets around single white dwarfs.

- Direct imaging. It has long been speculated that direct imaging should be the most promising method of detecting planets around white dwarfs owing to: (i) the better contrast between the white dwarf and the potential planet compared to main sequence stars; (ii) the orbital expansion of the planet 
during the red giant stage (Burleigh et al. 2002; Gould \& Kilic 2008). The only planetary-mass object around a single white dwarf was discovered via direct imaging with Spitzer: WD 0806-661B has a mass of $7 M_{J}$ at an orbital separation of $2500 \mathrm{AU}$ (Luhman et al. 2011). The most systematic search is the DODO (Degenerate Objects around Degenerate Objects) survey, which observed 29 white dwarfs with Gemini/NIRI and VLT/ISAAC, and their average upper limit is $\sim 8 M_{J}$ beyond $35 \mathrm{AU}$ (Hogan et al. 2011). There are also a few other direct imaging studies using HST/NICMOS (Debes et al. 2005a; Zinnecker \& Kitsionas 2008).

- Infrared excess. Because a white dwarf's luminosity peaks in the ultraviolet or optical, a cool companion can be easily detected as an infrared excess. Those searches started in the late $80 \mathrm{~s}$, and so far, only brown dwarf companions have been discovered (Zuckerman \& Becklin 1987; Maxted et al. 2006; Farihi et al. 2008; Kilic et al. 2009).

- Planetary transit. Faedi et al. (2011) studied 194 white dwarfs in the WASP survey and determined an upper limit of $10 \%$ for Jupiter-sized brown dwarfs and giant planets with orbital periods less than 0.1-0.2 d. Fulton et al. (2014) searched for eclipses around $\sim 1700$ white dwarfs in the PanSTARR1 field and found zero candidates, giving an upper limit of $0.5 \%$ for Jupiter-sized planets orbiting just outside of the Roche limit.

- White dwarf pulsation. Some white dwarfs have extremely stable pulsation modes, and the presence of a planet can cause a periodic change in the arrival time of the pulsation. Mullally et al. (2008) led a study of 15 such stable pulsators and identified one candidate: a $2 M_{J}$ mass planet in a $4.5 \mathrm{yr}$ orbit around GD 66. The planet hypothesis was dismissed later because of some other peculiar behavior in the pulsation of GD 66 (Mullally et al. 2009; Farihi et al. 2012; Hermes 2013). The upper limit from pulsation studies is typically a few Jupiter masses within 5 au of the white dwarf (Mullally et al. 2008).

In this paper, we present a pilot search for giant planets around a young white dwarf, GD 50. The data were taken in a science verification (sv) program with the new extreme-AO instrument SPHERE (Beuzit et al. 2008) at ESO's VLT. SPHERE includes the SAXO extreme adaptive optics system (Fusco et al. 2014; Petit et al. 2014), with a deformable mirror of $41 \times 41$ actuators, and four control loops (tip-tilt, high-orders, near-infrared differential tip-tilt, and pupil stabilization).

\section{Observation and data reduction}

\subsection{Target selection}

GD 50 (WD 0346-011) is a young nearby ultramassive hydrogen atmosphere white dwarf $\left(m_{V}=14.0, m_{R}=14.2, m_{H}=14.9\right)$. Its formation scenario has puzzled many astronomers. Dobbie et al. (2006) performed a complete astrometric and spectroscopic analysis and concluded that it has evolved as a single star and is an escaping white dwarf from the Pleiades open cluster. By fitting the photometric and spectroscopic data, they derived the effective temperature, surface gravity, and distance of GD 50 to be $T=41550 \mathrm{~K}, \log g=9.15$, and $d=31 \mathrm{pc}$. This corresponds to a white dwarf with a mass of $1.264 M_{\odot}$ and a total age of $125 \mathrm{Myr}$, which is consistent with the age of Pleiades. According to the initial-final mass relation in Williams et al. (2009), it had an initial mass of $7 M_{\odot}$, making its progenitor a B-type star.

GD 50 was chosen as our target for the SPHERE sv program due to its youth and close proximity. At $125 \mathrm{Myr}$, potential planets can still be quite hot and thus detectable. In addition, GD 50 has been observed with Spitzer/IRAC, and its $3 \sigma$ upper limit of $\sim 4 M_{J}{ }^{1}$ is among the best constraints from Spitzer searches (Farihi et al. 2008). A side goal of the project is to compare the performance of SPHERE with other instruments for detecting substellar objects around white dwarfs.

\subsection{Observation}

GD 50 was first observed on December 5, 2014. The data were not used for the analysis because of the poor observing conditions. The observation was repeated on February 1, 2015 with seeing around $11^{\prime \prime} 0$, a moon distance of $46^{\circ}$ (89\% illumination), and a coherence time of $\sim 5 \mathrm{~ms}$. The InfraRed Dual-band Imager and Spectrograph (IRDIS; Dohlen et al. 2008) was used in classical imaging (CI) mode in broadband $H$. The observations were made in pupil-stabilized mode, which is ideal for PSF subtraction, because the pupil and PSF stay fixed while the field rotates with respect to the detector. The integration time was $64 \mathrm{~s}$ per exposure and the observing block consisted of a $4 \times 4$ dither pattern with three exposures at each position, giving a total onsource time of $3072 \mathrm{~s}$.

\subsection{Data reduction}

The raw images were first corrected for flatfield errors and bad pixels. The white dwarf was unsaturated in all the images. Since no coronagraph was used, we were able to align the star on all images with subpixel accuracy. Owing to the faintness of the star, the stellar halo is weak, and there are hardly any speckles. Thus there is no need for PSF-subtraction. All images were derotated to align north up and median-combined. Following Wahhaj et al. (2013), a filtering process was applied to the median-combined image to isolate spatial features of $\sim 50$ mas in size, the diffraction limit in the $H$ band. This filter removes azimuthally extended features, such as the stellar halo, and improves the sensitivity to point sources for separations less than 0.3 . The signal-to-noise map is shown in Fig. 1.

Then we calculated the $5 \sigma$ contrast curve or the sensitivity limit relative to the primary. The peak intensity (star peak) at the stellar centroid was estimated by cubic interpolation. The pixelto-pixel rms was then measured in annuli of five pixels radii centered on the star for all separations (radii) of interest. The $5 \sigma$ contrast was computed as ( function of the separation from the star. No sources except the white dwarf were detected, and the contrast curve is shown in Fig. 2.

To check the robustness of our sensitivity limit, we cut out a circular aperture with a radius of four pixels around the star from each basic reduced image and reinserted it at the $5 \sigma$ contrast limit at several separations and position angles in the individual images. All the images were derotated and median-combined again. As shown in Fig. 1, all the inserted companions are recovered in the median-combined image. We have achieved $5 \sigma$ contrasts of $6.2,7.8,8,8.1$, and $8.25 \mathrm{mag}$ at $0.2,0 .{ }^{\prime} 3,0 .{ }^{\prime} 4,0.5$, and $0{ }^{\prime} 6$, respectively. In the background-limited region, the $5 \sigma$ limit corresponds to an apparent magnitude in $H$ band of $m_{H}=23.15$ (absolute magnitude $M_{H}=20.7$ ).

1 Farihi et al. (2008) report a $3 \sigma$ upper limit of $3 M_{J}$ assuming a total age of $100 \mathrm{Myr}$ for GD 50. To make the results directly comparable to our SPHERE observation, we adopted the revised age of $125 \mathrm{Myr}$ and the updated BT-Settl model grid (Allard 2014). Our updated Spitzer upper limit is $4.2 M_{J}$ for GD 50 . 


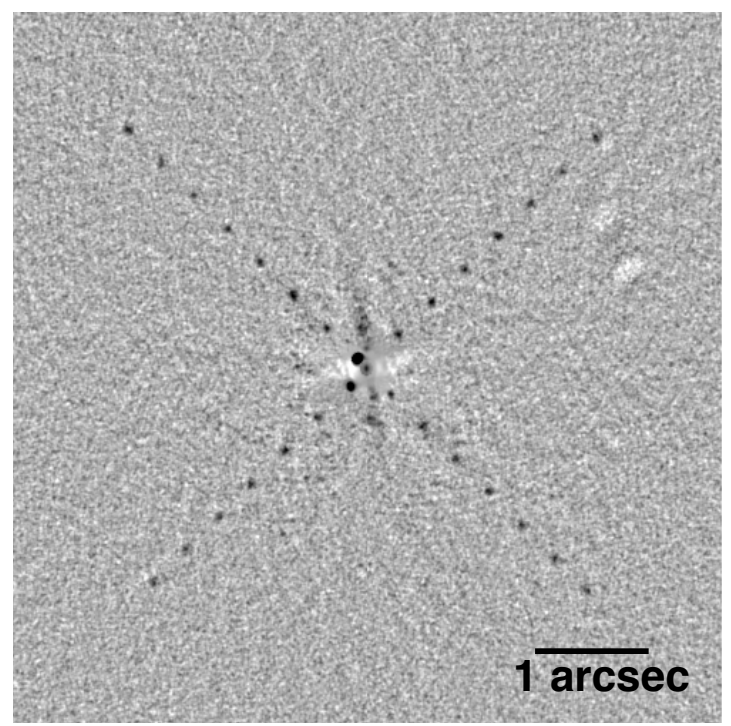

Fig. 1. Signal-to-noise map (i.e., reduced image divided by a noise map derived from it, see Sect. 2.3). The map has been smoothed using a Gaussian of FWHM 1.5 pixels. No astronomical point-like features were detected. In addition, we recovered artificial sources inserted into the raw data at the $5 \sigma$ contrast level at separations ranging from 100 mas to 3 arcsec with increments of 100 mas (X-shaped pattern of sources). The field of view displayed here is 6.'25 by 6.'25. Counts are displayed on linear scale and inverted (dark corresponds to high counts). North is up. The central white dwarf is not visible in this map because of image processing.

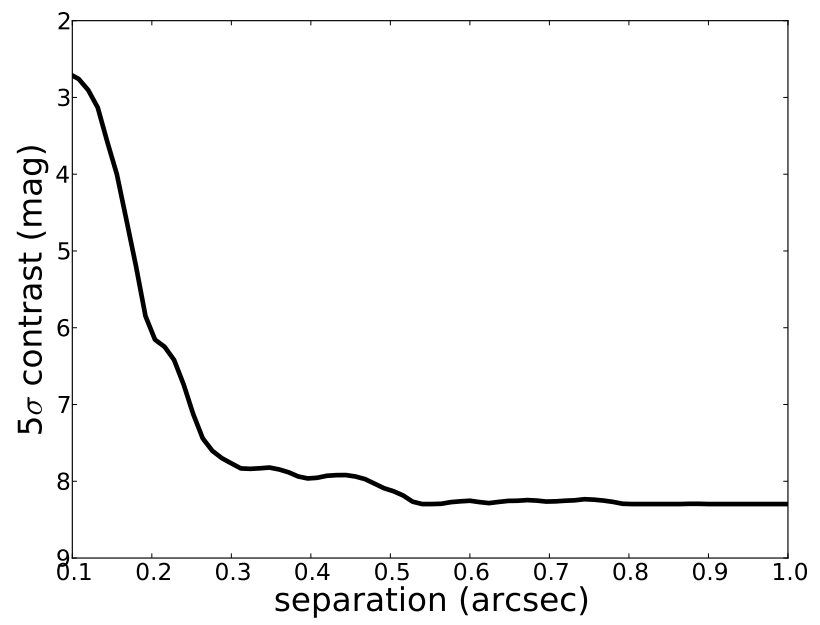

Fig. 2. $5 \sigma$ contrast curve for GD 50. A $5 \sigma$ contrast of $6.2,7.8,8.0$, 8.1, and 8.25 mags has been reached at 200 mas, 300 mas, 400 mas, 500 mas, and 600 mas, respectively.

\section{Discussion}

\subsection{Upper limits on planetary mass companions of GD 50}

To convert our detection limit to planetary mass, we used the BT-Settl models from Allard (2014). We took the projected separation as the orbital distance from the white dwarf and did not correct for the inclination effect. As shown in Fig. 3, we put an upper limit on the planetary mass of $4.0 M_{J}, 2.9 M_{J}$, and $2.8 M_{J}$ at $6.2 \mathrm{au}, 12.4 \mathrm{au}$, and 18.6 au and beyond, respectively.

Our upper limit corresponds to a limit of $2.8 M_{J}$ beyond 3.4 au during the main-sequence stage of the star. Depending on the metallicity, a $7 M_{\odot}$ star could reached a maximum radius of about 6.5 au during the post main-sequence evolution

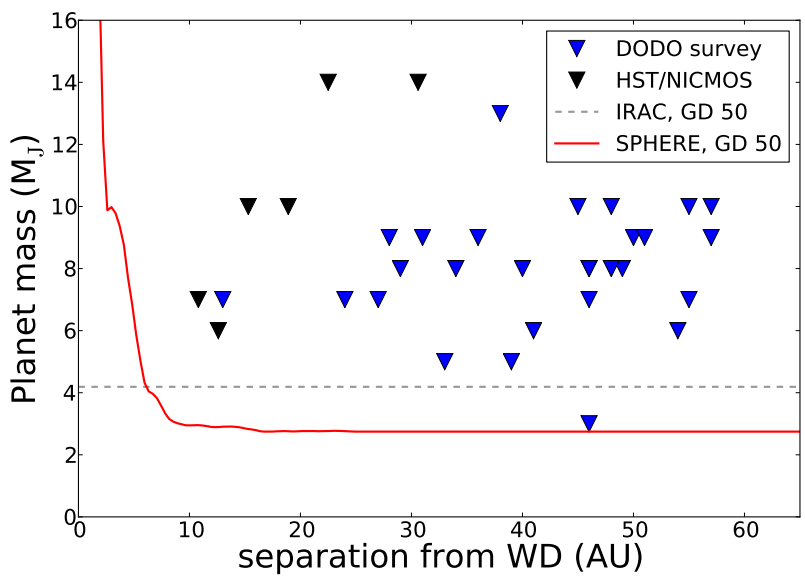

Fig. 3. Upper limits from previous imaging surveys (DODO survey from Hogan et al. 2011, HST/NICMOS studies from Debes et al. 2005a) for substellar objects around white dwarfs. The only positive detection so far is WD 0806-661B, but it is not shown because the separation is 2500 au (Luhman et al. 2011). We also include the upper limit on GD 50 from Spitzer/IRAC studies (Farihi et al. 2008). GD 50 is the most distant white dwarf in the figure, and yet our limit on GD 50 is still the most stringent.

(Veras et al. 2013), so any planets within 6.5 au were possibly destroyed. Therefore, it is unlikely that there are any giant planets more massive than $2.8 M_{J}$ around GD 50 now. For the surviving planets, their orbits will expand by a factor of about 7/1.264, which will be at least 36 au from GD 50. However, tidal interaction between the planet and the extended envelope of the AGB star can counteract the orbital expansion (Mustill \& Villaver 2012). Furthermore, if multiple planets were present, mutual interactions could scatter planets inward during the white dwarf stage (Veras \& Gänsicke 2015). As a result, some planets might end up having a much smaller semi-major axis. In addition, no heavy elements have been detected in the atmosphere of GD 50, though optical observations are not very sensitive to heavy elements at high stellar temperatures (Koester et al. 2009). GD 50 has a temperature of $41550 \mathrm{~K}$, and the abundance upper limits are not very constraining.

In Fig. 3, we compare the upper limit on GD 50 with all previous imaging searches for substellar objects around white dwarfs. Our upper limit on GD 50 is the most stringent, in terms of both mass and separation. For example, HST/NICMOS has an inner working angle of $0 . ' 9$ with the coronagraph (Debes et al. 2005a), which for GD 50 corresponds to 28 au in Fig. 3.

\subsection{Future observational prospects}

GD 50 is not representative of nearby white dwarfs - it is particularly young. Now we investigate the performance of SPHERE compared to other instruments for a generic white dwarf within $20 \mathrm{pc}$, with an absolute magnitude $M_{V}=12.0$ and $M_{H}=12.0$ and a total age of $2 \mathrm{Gyr}$ (Holberg et al. 2008). Since there are no BT-Settl models available for low-mass planets at 2 Gyr, AMESCOND models are used instead (Allard et al. 2001). As shown in the lefthand panel of Fig. 4, for nearby white dwarfs (within $\sim 30 \mathrm{pc}$ ), Spitzer photometric observation is limited by the calibration uncertainty of approximately $15 \%(3 \sigma)$; as a consequence, the Spitzer upper limit to planet mass remains the same regardless of the white dwarf distance. This is not the case for SPHERE because a potential planet would be spatially disentangled from the star outside an angular separation of $\sim 0^{\prime} 2$, about 
2 au at 10 pc. Resolved imaging with Spitzer can be more sensitive to lower mass objects, but at a much larger orbital separation (e.g., WD 0806-661B in Luhman et al. 2011). Spitzer observations become background-limited for more distant white dwarfs (beyond $30 \mathrm{pc}$ ), and all those white dwarfs are typically too faint for SPHERE. For nearby white dwarfs, resolved imaging with SPHERE is more sensitive to close-in low mass objects than photometric observations. There are $\sim 50$ white dwarfs that are brighter than $14.5 \mathrm{mag}$ in $R$ band and that are observable with SPHERE (Holberg et al. 2008). They are prime targets for future searches for planetary systems with SPHERE.

In the righthand panel of Fig. 4, we show the performance of SPHERE compared to other direct imaging instruments ${ }^{2}$. NICMOS and NACO have a much bigger inner working angle. The difference between NACO, HST, and SPHERE in the background-limited regime is minor. The real improvement of SPHERE over other instruments is in the region that is less than 1.'0. For a typical white dwarf within 20 pc, this corresponds to within $20 \mathrm{au}$ from the star. This region is particularly interesting because it can constrain models of the survival of planetary systems during the AGB phase.

Most white dwarfs have a progenitor mass between 2 and $5 M_{\odot}$. Searching for planets around white dwarfs directly probes this intermediate-mass region, which is very difficult with radial velocity and transit methods (Galland et al. 2005). Recently, Barber et al. (2014) have discovered a $1.04 M_{\odot}$ white dwarf (progenitor mass $5.4 M_{\odot}$ ) with a dust disk from tidally disrupted asteroid(s) - the first confirmation that massive stars can have planetary systems.

\section{Summary}

We have presented a pilot study with the extreme-AO instrument SPHERE to search for giant planets around a young white dwarf, GD 50. We excluded any substellar objects above $4.0 \mathrm{M}_{J}$, $2.9 M_{J}$, and $2.8 M_{J}$ at $6.2 \mathrm{au}(0.2), 12.4 \mathrm{au}\left(0 .{ }^{\prime} 4\right)$, and at $18.6 \mathrm{au}$ $(0.6)$ and beyond, respectively, which are among the best constraints compared to all previous studies. We further showed that SPHERE is the best instrument available to search for close-in substellar objects around nearby white dwarfs.

Acknowledgements. The authors thank an anonymous referee for useful comments. We thank France Allard for discussing various giant planet models and Monika Petr-Gotzens for useful discussions of the manuscript. We thank Carl Melis and Jay Farihi for helpful suggestions. We thank the SPHERE science verification team for performing the observations.

\section{References}

Allard, F. 2014, in IAU Symp. 299, eds. M. Booth, B. C. Matthews, \& J. R. Graham, 271

Allard, F., Hauschildt, P. H., Alexander, D. R., Tamanai, A., \& Schweitzer, A. 2001, ApJ, 556, 357

Barber, S. D., Kilic, M., Brown, W. R., \& Gianninas, A. 2014, ApJ, 786, 77
Beuzit, J.-L., Feldt, M., Dohlen, K., et al. 2008, SPIE Conf. Ser., 7014, 18 Bonsor, A., Mustill, A. J., \& Wyatt, M. C. 2011, MNRAS, 414, 930 Burleigh, M. R., Clarke, F. J., \& Hodgkin, S. T. 2002, MNRAS, 331, L41 Charpinet, S., Fontaine, G., Brassard, P., et al. 2011, Nature, 480, 496 Cumming, A., Butler, R. P., Marcy, G. W., et al. 2008, PASP, 120, 531 Debes, J. H., Sigurdsson, S., \& Woodgate, B. E. 2005a, ApJ, 633, 1168 Debes, J. H., Sigurdsson, S., \& Woodgate, B. E. 2005b, AJ, 130, 1221 Debes, J. H., Walsh, K. J., \& Stark, C. 2012, ApJ, 747, 148

Dobbie, P. D., Napiwotzki, R., Lodieu, N., et al. 2006, MNRAS, 373, L45 Dohlen, K., Langlois, M., Saisse, M., et al. 2008, SPIE Conf. Ser., 7014, 3 Faedi, F., West, R. G., Burleigh, M. R., Goad, M. R., \& Hebb, L. 2011, MNRAS, 410, 899

Farihi, J., Becklin, E. E., \& Zuckerman, B. 2008, ApJ, 681, 1470

Farihi, J., Subasavage, J. P., Nelan, E. P., et al. 2012, MNRAS, 424, 519

Frewen, S. F. N., \& Hansen, B. M. S. 2014, MNRAS, 439, 2442

Fulton, B. J., Tonry, J. L., Flewelling, H., et al. 2014, ApJ, 796, 114

Fusco, T., Sauvage, J.-F., Petit, C., et al. 2014, in SPIE Conf. Ser., 9148, 1

Galland, F., Lagrange, A.-M., Udry, S., et al. 2005, A\&A, 443, 337

Gänsicke, B. T., Marsh, T. R., Southworth, J., \& Rebassa-Mansergas, A. 2006, Science, 314, 1908

Gänsicke, B. T., Koester, D., Farihi, J., et al. 2012, MNRAS, 424, 333

Gettel, S., Wolszczan, A., Niedzielski, A., et al. 2012, ApJ, 745, 28

Gould, A., \& Kilic, M. 2008, ApJ, 673, L75

Hermes, J. J. 2013, Am. Astron. Soc. Meet. Abstr., 221, 424.04

Hogan, E., Burleigh, M. R., \& Clarke, F. J. 2011, in AIP Conf. Ser. 1331, eds. S. Schuh, H. Drechsel, \& U. Heber, 271

Holberg, J. B., Sion, E. M., Oswalt, T., et al. 2008, AJ, 135, 1225

Johnson, J. A., Clanton, C., Howard, A. W., et al. 2011, ApJS, 197, 26

Jura, M. 2003, ApJ, 584, L91

Jura, M. 2008, AJ, 135, 1785

Jura, M., \& Young, E. D. 2014, Ann. Rev. Earth Plan. Sci., 42, 45

Jura, M., Farihi, J., \& Zuckerman, B. 2007, ApJ, 663, 1285

Jura, M., Xu, S., Klein, B., Koester, D., \& Zuckerman, B. 2012, ApJ, 750, 69

Kilic, M., Gould, A., \& Koester, D. 2009, ApJ, 705, 1219

Klein, B., Jura, M., Koester, D., Zuckerman, B., \& Melis, C. 2010, ApJ, 709, 950

Koester, D. 2009, A\&A, 498, 517

Koester, D., Voss, B., Napiwotzki, R., et al. 2009, A\&A, 505, 441

Koester, D., Gänsicke, B. T., \& Farihi, J. 2014, A\&A, 566, A34

Luhman, K. L., Burgasser, A. J., \& Bochanski, J. J. 2011, ApJ, 730, L9

Macintosh, B., Graham, J. R., Ingraham, P., et al. 2014, Proc. National Academy of Science, 111, 12661

Maxted, P. F. L., Napiwotzki, R., Dobbie, P. D., \& Burleigh, M. R. 2006, Nature, 442, 543

Mullally, F., Winget, D. E., De Gennaro, S., et al. 2008, ApJ, 676, 573

Mullally, F., Reach, W. T., De Gennaro, S., \& Burrows, A. 2009, ApJ, 694, 327

Mustill, A. J., \& Villaver, E. 2012, ApJ, 761, 121

Nordhaus, J., \& Spiegel, D. S. 2013, MNRAS, 432, 500

Petit, C., Sauvage, J.-F., Fusco, T., et al. 2014, in SPIE Conf. Ser., 9148, 0

Setiawan, J., Klement, R. J., Henning, T., et al. 2010, Science, 330, 1642

Silvotti, R., Schuh, S., Janulis, R., et al. 2007, Nature, 449, 189

Veras, D., \& Gänsicke, B. T. 2015, MNRAS, 447, 1049

Veras, D., Mustill, A. J., Bonsor, A., \& Wyatt, M. C. 2013, MNRAS, 431, 1686

Wahhaj, Z., Liu, M. C., Biller, B. A., et al. 2013, ApJ, 779, 80

Williams, K. A., Bolte, M., \& Koester, D. 2009, ApJ, 693, 355

Xu, S., \& Jura, M. 2012, ApJ, 745, 88

Xu, S., Jura, M., Koester, D., Klein, B., \& Zuckerman, B. 2014, ApJ, 783, 79

Zinnecker, H., \& Kitsionas, S. 2008, in Extreme Solar Systems, eds. D. Fischer, F. A. Rasio, S. E. Thorsett, \& A. Wolszczan, ASP Conf. Ser., 398, 155

Zuckerman, B., \& Becklin, E. E. 1987, ApJ, 319, L99

Zuckerman, B., Melis, C., Klein, B., Koester, D., \& Jura, M. 2010, ApJ, 722, 725

Page 5 is available in the electronic edition of the journal at http://www . aanda.org

\footnotetext{
2 The performance of GPI is not shown because it has a limiting magnitude of $m_{I}=10$ (Macintosh et al. 2014). There are very few white dwarfs that are bright enough to be observed with GPI.
} 
S. Xu et al.: An extreme-AO search for giant planets around a white dwarf
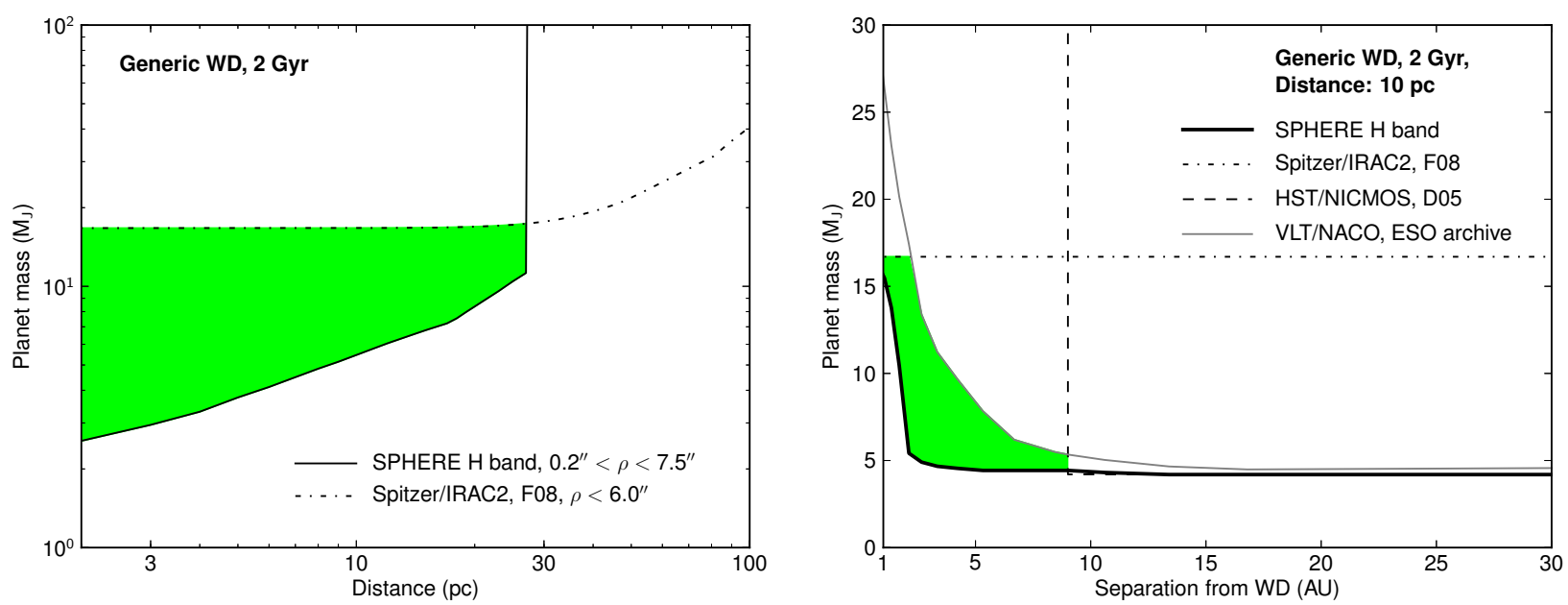

Fig. 4. Illustration of the performance of SPHERE compared to other instruments for a typical nearby white dwarf (age $\left.2 \mathrm{Gyr}, M_{V}=M_{H}=12\right)$. The green regions represent the parameter space for which SPHERE enables significant improvement over the other instruments. Left: sensitivity vs. distance of the white dwarf from the Sun. The quantity $\rho$ represents the angular separation that the instrument is sensitive to. The performance of SPHERE is extrapolated from our observation of GD 50. Because GD 50 is at the faintness limit, the actual performance on a brighter target would be better. The vertical cut-off in the SPHERE curve near $30 \mathrm{pc}$ is artificial and assumes that a star fainter than $m_{V}=14.5$ cannot be observed because of the limiting magnitude of the AO. For nearby bright white dwarfs, resolved imaging is more sensitive to low-mass objects than photometric observations. Right: contrast curves for the same WD at a distance of $10 \mathrm{pc}$. The NACO curve is estimated from archival data (program ID: 079.D-0561(A), PI: Radiszcz) with a similar integration time as SPHERE (3000 s). The HST/NICMOS limit was extrapolated from Debes et al. (2005b) with a typical exposure time of $20 \mathrm{~min}$. The key point is that SPHERE has the unique capability to detect high-mass planets at 2-10 au from the white dwarf, which is not possible with any other instrument. 